\title{
El CRÉDITO BANCARIO COMO AR(1): EL CASO DE MÉXICO 1980-2003
}

\author{
Carlos Pulido.* \\ Tecnológico de Monterrey, Campus Ciudad de México \\ (Recibido 25 de junio 2004, aceptado 26 de agosto 2004)
}

\section{Resumen}

La literatura actual considera que el crédito bancario es un factor que incentiva el crecimiento económico. Para el caso de México, durante el período 1980-2003, se observa que el crédito -como proporción del PIB- muestra en general una trayectoria temporal constante o, si acaso, débilmente decreciente. Esta evidencia indica que la proporción crédito-PIB es una serie de tiempo que podría ser estacionaria y modelada con un proceso autorregresivo de orden p $(A R(p))$. El presente trabajo tiene como objeto mostrar que la serie es adecuadamente modelada con un $\mathrm{AR}(1)$ y que ésta representación puede ser sustentada consistentemente con un modelo elemental de programación dinámica.

\section{Abstract}

Current literature considers banking credit as a factor that promotes economic growth. For the Mexican case, in the period 1980-2003, we observe that credit -relative to GDP- reflects a constant temporal path or, at most, a non pronounced increasing temporal path. This evidence indicates that ratio credit-GDP is a time series that could be stationary and modeled with a pth-order autorregressive process $(\operatorname{AR}(p))$. This paper shows that the series is well modeled with an $\mathrm{AR}(1)$ an that this representation is consistent with an elemental dynamic programming model.

Clasificación JEL: C22, O47, G10

Palabras clave: Series de Tiempo, Crecimiento Económico, Sistema Financiero

* Centro de Investigación en Finanzas, Tecnológico de Monterrey, Campus Ciudad de México, Calle del Puente 222, Aulas 3, Cuarto Piso, Col. Ejidos de Huipulco, Del. Tlalpan, 14380, México, D.F. Correo electrónico: carlospulido780@yahoo.com.mx 


\section{Introducción}

La literatura económica considera que en general existen cuatro insumos que intervienen en la producción de cualquier bien: trabajo, capital físico, capital humano y recursos naturales. Sin embargo, la literatura del crecimiento económico de la última década se enfocó intensamente en otro factor productivo: el crédito bancario. Dos ejemplos de esta literatura son Bencivenga y Smith (1991), quienes estudiaron la relación teórica entre crecimiento y crédito, y Benhabib y Spiegel (2001), quienes analizaron la relación empírica entre las dos variables. Las conclusiones obtenidas en ambos casos son que el crédito bancario influye positivamente sobre el crecimiento económico, porque permite acumular más capital físico privado y público, y que la relación crédito-crecimiento es estadísticamente significativa.

Uno de los indicadores de crédito bancario que emplea la literatura para vincular a éste con el crecimiento es la razón crédito total-PIB. Es decir, el volumen de préstamos realizados por la banca comercial a empresas, familias y gobierno como proporción del producto. En particular, se considera que una razón alta y creciente es un indicador confiable de que el sistema financiero de una economía es desarrollado.

El sistema financiero mexicano actual es considerado en proceso de desarrollo. Dos eventos importantes que contribuyeron a esta definición sucedieron en los períodos 1982-1988 y 1989-1994. Durante el primer período se presentó una represión financiera: el crédito total disminuyó drásticamente y fue prácticamente asignado al gobierno, en detrimento de la acumulación de capital privado. Lo contrario sucedió durante el segundo período en el que existió una expansión financiera: el crédito total aumentó y fue canalizado significativamente a empresas y familias. Sin embargo, esta asignación fue realizada sin monitoreo suficiente por lo que, ante la crisis económica de 1995, los bancos cayeron en corrida bancaria frente a la imposibilidad de repago por parte de muchos deudores. Para que esta situación no volviera a ocurrir, se crearon instituciones públicas (FOBAPROA e IPAB) que a partir de ese momento regulan más estrictamente el crédito.

Gráfica 1. Serie de Tiempo de la Razón Crédito Bancario-PIB Crédito bancario/PIB (México, 19800-2003)

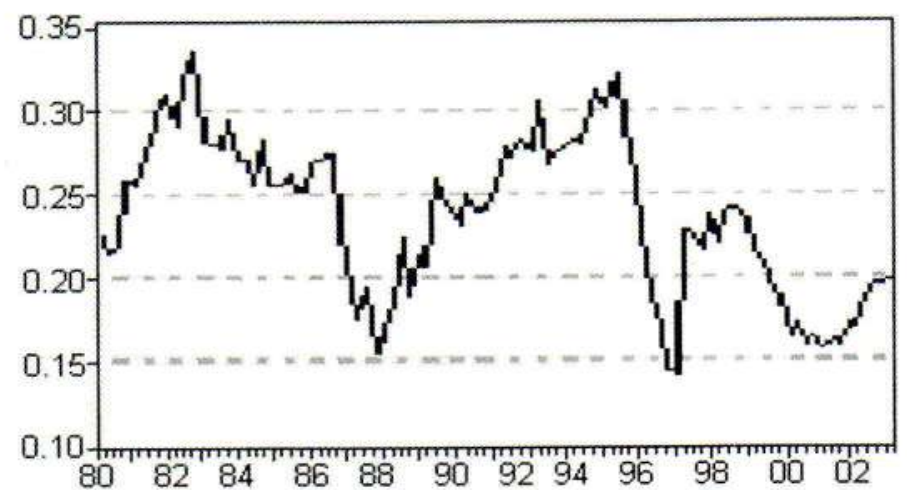


Formalmente, la evolución de la razón crédito total-PIB para el caso de México durante los años 1980-2003 se presenta en la Gráfica 1. La Gráfica 1 indica dos hechos. En primer lugar, la mayoría de los valores de la serie se encuentran dentro del intervalo $(0.15,0.3)$. En segundo lugar, a pesar de que la serie es no monótona, muestra en general una trayectoria temporal débilmente decreciente. Específicamente, la serie presenta una tendencia creciente para el período de 1988-1994 (años de expansión financiera privada) pero decreciente para los períodos 1982-1988 (años de represión financiera privada) y 1995-2003 (años de fuerte monitoreo en la asignación de créditos).

Desde el punto de vista del análisis de series de tiempo, el hecho de que prácticamente todos los valores de la serie crédito total-PIB se encuentren en un determinado intervalo no amplio es una primera aproximación de que la serie es estacionaria. Asimismo, el hecho de que la serie crédito total-PIB muestre una tendencia decreciente y débil es evidencia de que esta serie podría ser representada por un modelo autorregresivo cuyo(s) coeficiente(s) represente(n) la convergencia estocástica de la serie hacia algún valor.

Este trabajo tiene como objeto verificar estas dos afirmaciones, mediante la construcción de un modelo de series de tiempo que describa adecuadamente la evolución temporal de la serie de tiempo crédito total-PIB. Para tal efecto, el trabajo se divide en dos partes. En la primera parte, se plantea un modelo teórico del cual se deriva que, como resultado de la maximización intertemporal de beneficios esperados por parte de los agentes que emiten crédito bancario (los intermediarios financieros), la razón crédito total-PIB sigue un comportamiento autorregresivo de orden uno, el cual puede ser convergente, explosivo, o de caminata aleatoria dependiendo del valor de ciertos parámetros. En la segunda parte, se verifica esta conclusión mediante la construcción de un modelo univariado de series de tiempo, del cual se analizan sus supuestos básicos. Finalmente, se ofrecen las conclusiones.

\section{Modelo teórico}

En esta sección, se propone un modelo simple en el que existen intermediarios financieros cuyo objetivo es maximizar su flujo intertemporal de beneficios. De aquí se obtendrá la ecuación que dicte el comportamiento temporal de la razón crédito total-PIB. Con este propósito, se ofrecen a continuación los supuestos y el desarrollo del modelo.

\subsection{Supuestos}

Sea una economía con un horizonte temporal discreto e infinito, en la que existen intermediarios financieros que se dedican a asignar préstamos al resto de los agentes. Sea $B_{t}$ el nivel de créditos y $Y_{t}$ el nivel de producción de la economía. Supóngase que $b_{t} \equiv B_{t} / Y_{t}$-el nivel de crédito como proporción del producto- es la variable de control relevante para los intermediarios. La cantidad de crédito que se demanda a éstos depende inversamente de la tasa de interés activa $r_{t}^{a}$. Formalmente, la demanda por crédito viene dada por:

$$
r_{t}^{a}=1-\frac{a}{2} b_{t}
$$


donde $a$ es un parámetro positivo. Por simplicidad, considérese que los intermediarios no enfrentan costos de ningún tipo. En este caso su función de beneficios en $t, \pi_{t}$, es:

$$
\pi\left(b_{t}\right)=b_{t}-\frac{a}{2} b_{t}^{2} .
$$

Supóngase que los préstamos realizados por los intermediarios provienen exclusivamente de los depósitos $\mathrm{d}_{t}$ que se realizan en ellos. Así, el objetivo de los intermediarios es maximizar su flujo intertemporal de beneficios esperados, sujeto a su restricción de recursos. Es decir,

$$
\begin{aligned}
& \operatorname{Max} E_{t} \sum_{t=0}^{\infty} \beta_{t} \pi\left(b_{t}\right) \\
& \text { s.a. } \sum_{t=0}^{\infty} \frac{b_{t}}{R_{t}}=\sum_{t=0}^{\infty} \frac{d_{t}}{R_{t}},
\end{aligned}
$$

donde $E_{t}$ es el valor esperado en $t, \beta \equiv 1 /(1+\rho)$ es el factor de descuento intertemporal ( $\rho$ es la tasa subjetiva de descuento) y, $R_{t} \equiv \Pi_{s=0}^{t}\left(1+r_{s}\right.$ ) es la tasa de interés de mercado no necesariamente igual a la tasa activa.

\subsection{Desarrollo}

La ecuación de Euler del problema (3) viene dada por:

$$
1-a b_{t}=\beta R_{t}-a \beta R_{t} E_{t}\left(b_{t+1}\right) .
$$

Considere que $u_{t}$ es un proceso de ruido blanco con media cero, definido como $u_{t}=b_{t}-E_{t-1}\left(b_{t}\right)$. En este caso, se tiene que $E_{t}\left(b_{t+1}\right)=b_{t+1}-u_{t+1}$. Al sustituir esta expresión en (4), resolver para $b_{t+1}$ y asumir que $R_{t}$ es constante $\left(R_{t}=R\right)$, resulta:

$$
b_{t+1}=\frac{\beta R-1}{\alpha \beta R}+\frac{1}{\beta R} b_{t}+u_{t+1} .
$$

Obsérvese que (5) es un Proceso Autorregresivo de Primer Orden (AR(1)), el cual será estacionario (tendrá convergencia estocástica) si el valor absoluto de $1 / \beta R$ es menor que uno. Para que esto suceda, se requiere que $\rho<r$, y esto será más probable cuando la tasa de interés sea alta y/o el intermediario financiero valúe de un modo importante sus beneficios futuros, no sólo los presentes. Finalmente, la ecuación de Euler se puede rescribir como:

$$
E_{t}\left(b_{t+1}\right)=\frac{\beta R-1}{a \beta R}+\frac{1}{\beta R} b_{t} .
$$

Dada (6) y la condición de convergencia se tiene que, para determinados valores de $\beta, R$ y $a$, el proceso estocástico $b_{t}$ puede ser una supermartingala, una martingala o una submartingala. En particular, para valores pequeños de $b_{t}$, el proceso es una submartingala $\left(E_{t}\left(b_{t}+1\right)>b_{t}\right)$; para valores grandes de $b_{t}$, el proceso es una supermartingala $\left(E_{t}\left(b_{t}+1\right)<b_{t}\right)$. Por último, cuando $b_{t}=1 / a$, el proceso es una martingala $\left(E_{t}\left(b_{t}+1\right)=b_{t}\right)$. Véase la Figura 1. 
Figura 1. El proceso $b_{t}$ contra $E\left(b_{t+1}\right)$.

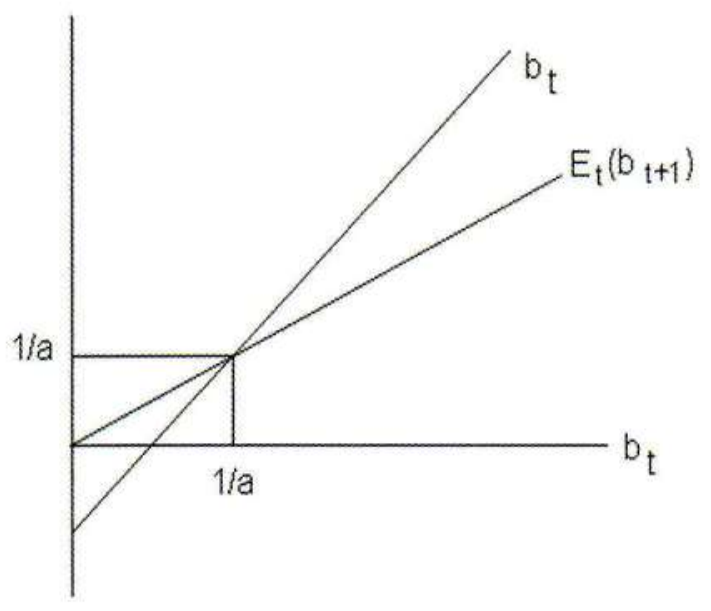

\section{Evidencia empírica}

En esta sección se construirá un modelo de serie de tiempo para la variable crédito bancario-PIB, y se verificará si este modelo coincide con aquel propuesto en la sección anterior. Esto es, si es un modelo AR(1). Por inspección visual de la Gráfica 1, aparentemente esta serie de tiempo podría ser débilmente estacionaria, debido a que no presenta una tendencia temporal monótona en algún sentido y a que prácticamente todos sus valores se encuentran en un determinado intervalo. Ahora se probará formalmente esta afirmación. Para tal efecto, se asumirá que, inicialmente, la razón crédito bancario-PIB es no estacionaria tanto en media como en varianza, en cuyo caso será necesario estabilizar la serie en ambos momentos antes de proponer modelos que describan su comportamiento en el tiempo. Siguiendo el criterio de Guerrero (1993), primero se estabilizará la varianza y después la media. Lo primero se logra transformando la serie original en otra serie, mientras que lo segundo se consigue cuando se toman las diferencias necesarias sobre la serie transformada para hacerla estacionaria. A continuación se muestran ambos procedimientos aplicados a la serie, la cual fue obtenida de las Estadísticas Financieras Internacionales (EFI) del FMI. La muestra seleccionada considera datos trimestrales del período 1980:1-2003:1 $1^{1}$. De este modo, se tienen $\mathrm{N}=93$ datos.

\subsection{Estabilización de varianza}

Para empezar, se reúnen todos los datos trimestrales en $H=23$ grupos, donde cada grupo tiene cuatro observaciones y representa un año desde 1980 hasta 2002 -la observación del primer trimestre del 2003 queda excluida. Posteriormente, de cada grupo se obtiene su media y desviación estándar. A partir de estas dos medidas se calcula el cociente:

$$
S_{h} / Z_{h}^{1-\lambda}, \quad h=1, \cdots, H,
$$

1 Para la obtención del crédito total y del PIB se consideraron las líneas 52A-52D y 99B, respectivamente, de las EFI. 
donde $Z_{h}$ y $S_{h}$ son la media y la desviación estándar, respectivamente, del grupo $h$ y $\lambda$ es la potencia a la que se transformará la serie original (por lo general, $\lambda$ se encuentra en el intervalo $(-2,2)$ ). Formalmente, la serie transformada $T\left(b_{t}\right)$ se define como:

$$
T\left(b_{t}\right)=b_{t}^{\lambda}
$$

De este modo, se busca que (7) sea constante para cada uno de los $H$ grupos, porque con ello se minimizará el coeficiente de variación tomado sobre los 23 grupos para una determinada $\lambda$. Al usar la función Solver (C) de Excel ${ }^{(C)}$, se concluye que el valor de la potencia que minimiza el coeficiente de variación es igual a 1.05. Por tanto, la serie transformada de la razón crédito bancario-PIB es $T\left(b_{t}\right)=b_{t}^{1.05}$. Sin embargo, esta transformación no es práctica. Dado que el coeficiente de variación es una función decreciente del valor de $\lambda$, se elegirá la transformación más sencilla $T\left(b_{t}\right)=b_{t}$. Es decir, la serie original. Obsérvese que esto confirma que la serie crédito-PIB presenta variabilidad homogénea. Los resultados de este procedimiento se encuentran en la Tabla A del Anexo.

\subsection{Estabilización de la media}

De acuerdo a la sección anterior, la serie transformada que se volverá estacionaria en nivel es $b_{t}$. Para tal efecto, se requiere obtener el grado de diferenciación óptimo para estabilizar su nivel. Aquí se propone un método para detectar ese grado: la función de autocorrelación muestral (FAC). Se sabe que una serie es estacionaria si su FAC converge rápidamente a cero, y viceversa. A continuación se grafica la FAC de la razón crédito bancario-PIB ( $k$ es el valor del rezago):

Gráfica 2. FAC para verificar estacionariedad

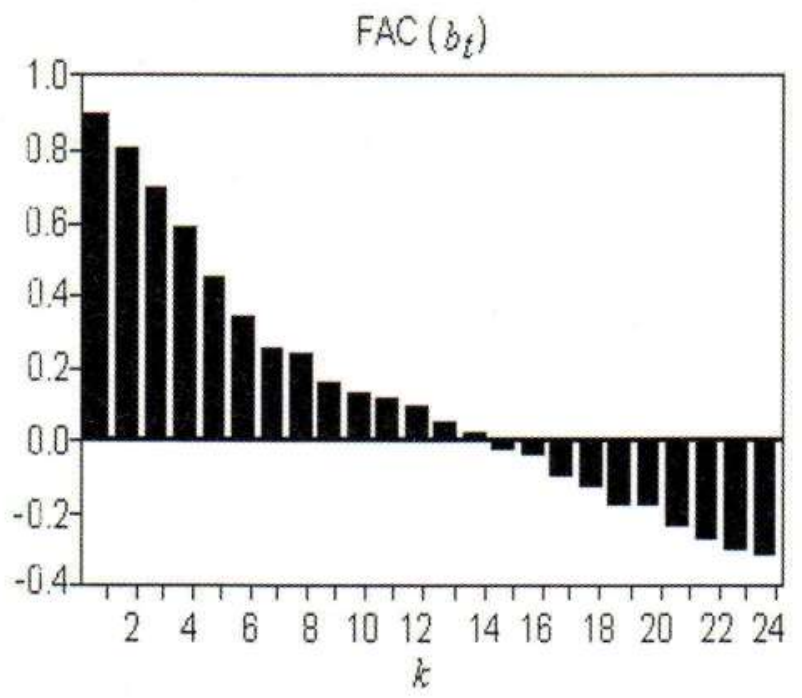


De acuerdo a la Gráfica 2, la FAC de la serie converge rápidamente a cero. En particular, del retraso ocho al veinte, la FAC se encuentra en el intervalo $(-0.2$, 0.2 ). Obsérvese que estos límites son pequeños. Por tanto, se concluye que la serie crédito bancario-PIB es estacionaria en nivel. Esto verifica que la serie no presenta una fuerte tendencia temporal creciente ni decreciente.

\subsection{Identificación del modelo}

Para identificar posibles modelos de series de tiempo que representen adecuadamente a la razón crédito-PIB, se usará tanto la FAC como la función de autocorrelación parcial (FACP) de la serie transformada, que en este caso es la serie original. Para tal efecto, se sabe que, si la serie se comporta como un proceso $\mathrm{AR}(\mathrm{p})$-que es el tipo de ecuación modelado en la primera parte- entonces debe cumplirse que la FAC converja a cero y que solamente las primeras "p" autocorrelaciones parciales sean distintas de cero. En la Gráfica 3, se presenta la FACP de la serie transformada.

Gráfica 3. FACP para verificar el orden del modelo AR

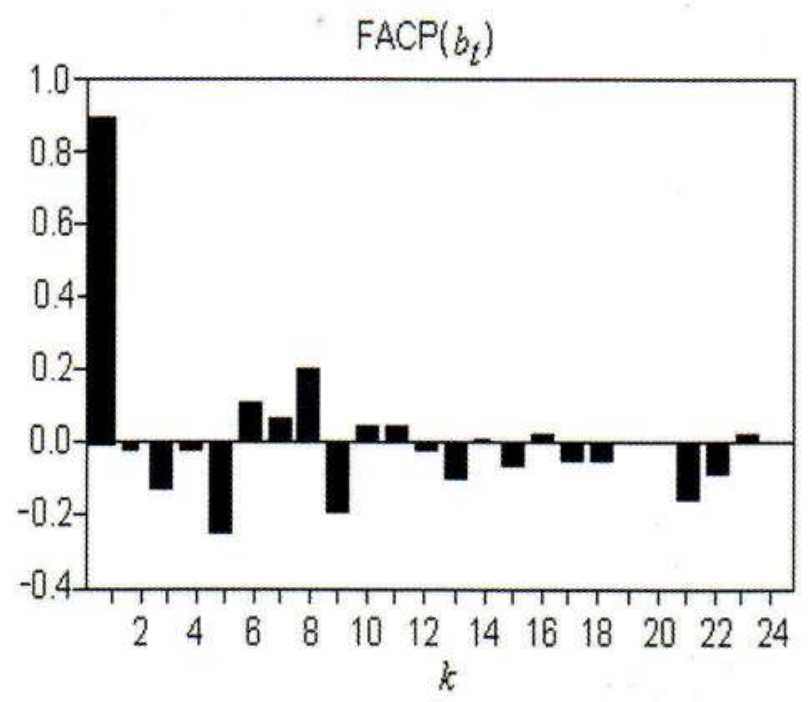

De acuerdo a la Gráfica 3 se observa que la serie del crédito bancario presenta una FACP en la que sólo la primera autocorrelación parcial es estadísticamente significativa. Líneas arriba se mencionó que la FAC de la serie converge rápido a cero. Por tanto, se concluye que la serie crédito bancario puede ser modelada mediante un AR(1). A continuación se realiza la estimación del modelo AR(1) mediante el método de mínimos cuadrados ordinarios (MCO). Los resultados son:

$$
b_{t+1}=0.2334+0.9020 b_{t}+u_{t},
$$

donde los estimadores son estadísticamente significativos al $1 \%$ (véase la Tabla $\mathrm{B}$ del Anexo). La ecuación (9) verifica lo mencionado anteriormente en el modelo teórico: el crédito bancario presenta, en general, una trayectoria temporal 
decreciente pero débil. Ambas características se comprueban con el coeficiente de $b_{t}(0.9020)$, el cual es menor a uno pero cercano a él. En el modelo teórico se planteó que un factor que incentiva a tener un coeficiente menor a uno es la existencia de tasas de interés altas, y esto es propio de economías con sistema financiero en proceso de desarrollo. México es un ejemplo de este tipo de sistema.

\subsection{Verificación de los supuestos del modelo}

Para detectar violaciones a los supuestos del modelo propuesto se realizará un análisis de residuales $s\left(e_{t}\right)$ del modelo debido a que, para un tamaño de muestra grande, esto equivale a analizar el proceso de ruido blanco. A continuación se presentan ocho supuestos para analizar:

\section{Supuesto 1: El error tiene media cero}

Verificación: Se calcula el estadístico $\left(m\left(e_{t}\right) / s\left(e_{t}\right)\right) \sqrt{(N-1)}$, el cual es función de la media $m\left(e_{t}\right)$ y de la desviación estándar $s\left(e_{t}\right)$ de los residuos. Si este estadístico es en valor absoluto menor a dos, entonces se concluye que el supuesto no se viola, y viceversa. En este caso, se tiene la Tabla 1:

Tabla 1. Verificación del supuesto media cero en el error

\begin{tabular}{|c|c|c|c|}
\hline Media & Desviación Estándar & Estadístico & Decisión \\
\hline$-2.03 \mathrm{E}-14$ & 0.0208 & $-9.2922^{-12}$ & No hay violación \\
\hline
\end{tabular}

Por tanto, se concluye que el valor esperado del error es cero.

\section{Supuesto 2: El error tiene varianza constante}

Verificación: Se graficará la serie de tiempo de los residuales para observar su variabilidad. En la Gráfica 4 se muestra la serie.

Gráfica 4. Verificación del supuesto varianza constante en el error Residuales del crédito bancario/PIB (México, 1980-2003)

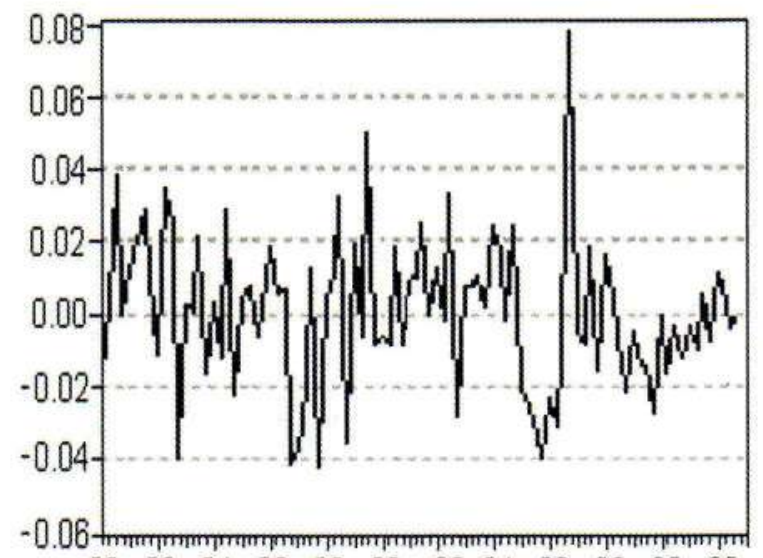

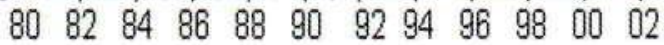


De acuerdo a la Gráfica 4 se observa que, con excepción de los residuales 1996:4 y 1989:3, todos los demás se encuentran dentro del intervalo (-0.04, 0.04). Por tanto, se concluye que la varianza del residual es constante.

\section{Supuesto 3: Los errores son mutuamente independientes}

Verificación: Se sabe que, si existe autocorrelación entre los errores, entonces éstos no serán independientes. Por tanto, se van a realizar dos pruebas sobre correlación serial de residuales. La primera consiste en obtener la FAC de los residuales para probar su significancia individual. La segunda consiste en calcular el estadístico Q de Ljung y Box para probar la significancia conjunta de las primeras $\mathrm{k}$ autocorrelaciones entre residuales. A continuación se presentan ambas pruebas mediante las Gráficas 5a y 5 b.

Gráfica 5a. Verificación del supuesto independencia en el error

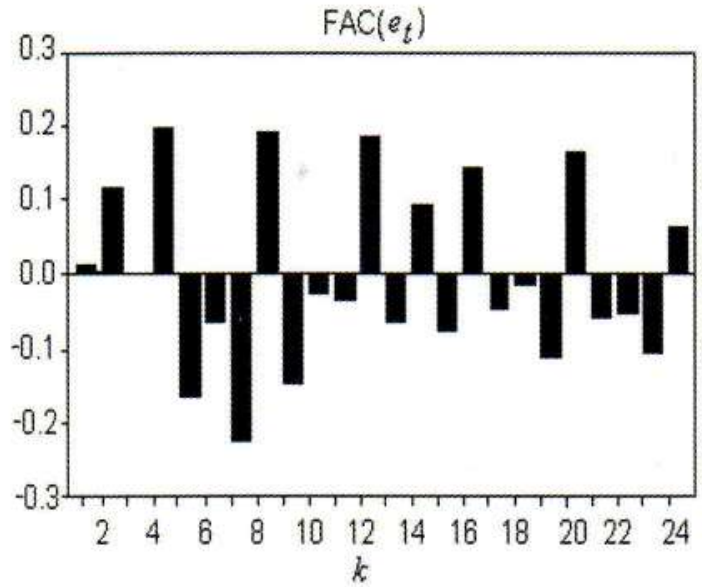

Gráfica 5b. Verificación del supuesto independencia en el error Valor-p del estadístico Q

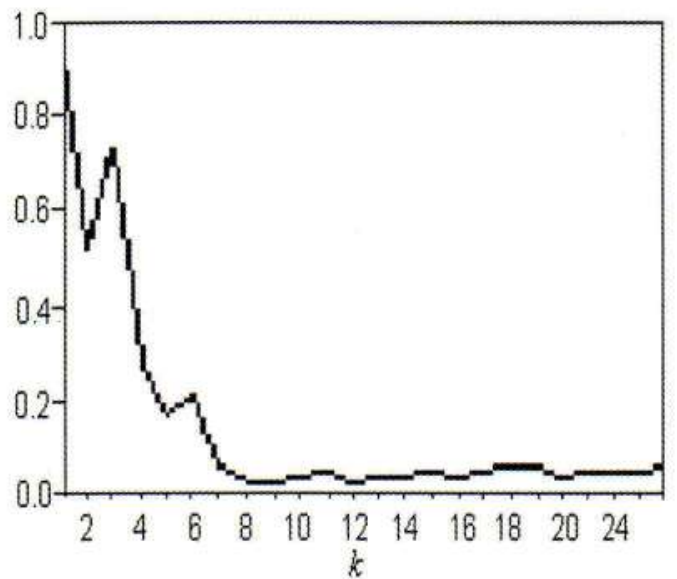


Para determinar la significancia individual se proponen las bandas de confianza $+/-2 / \sqrt{(N-1)}$. En este caso, el intervalo de confianza es $(-0.2096,0.2096)$. De acuerdo a la Gráfica 5a se observa que prácticamente ninguna autocorrelación sale fuera del intervalo. Por tanto, se concluye que cada una de las autocorrelaciones es estadísticamente igual a cero. Asimismo, con respecto al estadístico Q, la Gráfica 5b indica que, a pesar de que el valor-p asociado al estadístico es decreciente con respecto al número de retrasos, ningún valor-p es menor a 0.01 , por lo que se afirma con un nivel de significancia de $1 \%$ que el grupo de las primeras $k$ autocorrelaciones son iguales a cero. En conclusión, los errores son independientes entre sí.

\section{Supuesto 4: los errores se distribuyen normalmente}

Verificación: Se realizará la prueba de normalidad Jarque-Bera (JB). Para tal efecto, se presenta el histograma de los residuales, sus coeficientes de asimetría (CA) y curtosis (CC), el estadístico JB y su valor-p asociado.

Gráfica 6. Verificación del supuesto normalidad en el error Histograma de los residuales

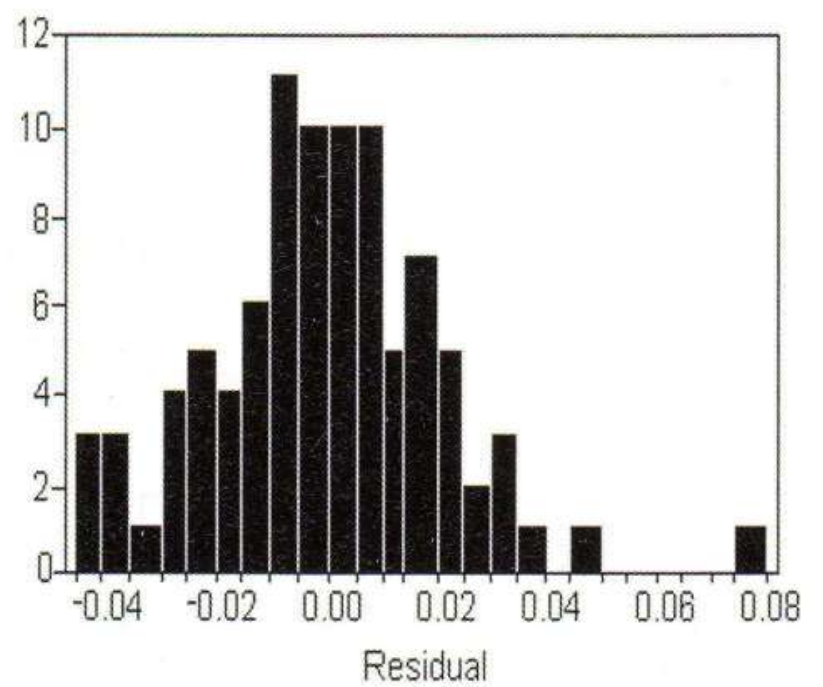

$$
\begin{gathered}
C A=0.4225 \\
C C=4.1620 \\
J B=7.9138 \\
\text { Valor }-\mathrm{p}=0.0191
\end{gathered}
$$


De acuerdo a la Gráfica 6, no se aprecia sesgo ni aplanamiento/elevación excesivos en la distribución de los residuales. Esto se confirma con los coeficientes de asimetría y curtosis: el primero es cercano a cero; el segundo es cercano a tres. En suma, el valor-p del estadístico JB indica que no se rechaza el supuesto de normalidad con un nivel de significancia de $1 \%$.

\section{Supuesto 5: No existen observaciones aberrantes en el modelo}

Verificación: Se considera que un residual cumple esta característica cuando se encuentra fuera del intervalo $\left(-3 s\left(e_{t}\right), 3 s\left(e_{t}\right)\right)$. De acuerdo a la Gráfica 4 , sólo la observación del trimestre 1996:4 satisface esto. Sin embargo, esta observación aberrante obedece a la presencia de un hecho inusual en esa fecha: la creación de una institución pública (FOBAPROA) que reguló la emisión de crédito a la economía. Por tanto, esta observación es resultado de un análisis de intervención exógena en la serie y, por ello, no se elimina de la serie estudiada.

\section{Supuesto 6: El modelo es parsimonioso}

Verificación: Cuando un modelo cumple esta característica, sus parámetros son necesarios para explicar el fenómeno y, por tanto, no pueden ser iguales a cero. De este modo, se construirá un intervalo de confianza sobre el estimador del parámetro y se verificará si el cero se encuentra dentro de dicho intervalo. El intervalo viene dado por $0.9020+-2(0.0463)$, donde 0.9020 es el valor del estimador y 0.0463 es su error estándar. Entonces, los límites inferior y superior del intervalo son $(0.8094,0.9940)$. Dado que el cero no es un posible valor que pueda tomar el parámetro, se concluye que el modelo es parsimonioso.

\section{Supuesto 7: El modelo es admisible}

Verificación: Debido a que este modelo es un $\mathrm{AR}(1)$, basta con inspeccionar si el parámetro estimado se encuentra dentro de la región admisible. En este caso, el valor del estimador es menor a uno $(0.9020)$, por lo que la raíz $x$ de la ecuación $1-0.9020 x=0$ se encuentra fuera del círculo unitario (es igual a 1.1086) y, por tanto, cumple la condición de estacionariedad en el modelo.

\section{Conclusiones}

Para el caso de México y durante el período 1980:1-2003:1, la serie de tiempo crédito bancario total-PIB mostró una tendencia temporal no monótona: presentó una tendencia creciente en el período 1989-1994, pero dos tendencias decrecientes en los períodos 1982-1988 y 1995-2003. Adicionalmente, casi todos los valores de la serie se encontraron dentro del intervalo estrecho $(0.15,0.3)$. A partir de esta información se proponen dos resultados. En primer lugar, el crédito total como proporción del PIB tendió a disminuir en el tiempo analizado. En segundo lugar, esta tendencia fue débil por lo que se afirma que el crédito fue -regularmente- entre $15 \%$ y $30 \%$ del producto.

Ambos resultados pueden ser justificados tanto teóricamente como empíricamente. Lo primero, mediante un modelo simple de optimización dinámica en el que los intermediarios financieros sólo buscan maximizar su flujo intertemporal esperado de beneficios. Lo segundo, a través de un modelo univariado de series de tiempo tipo $\mathrm{AR}(1)$. Ambos modelos coinciden entre sí y, mientras que el modelo teórico ofrece tres posibilidades sobre el comportamiento de 
la razón crédito total-PIB como proceso estocástico (supermantigala, martingala o submartingala), el modelo empírico satisface los supuestos básicos que fundamentan a un modelo de series de tiempo.

\section{Anexo}

En seguida se presentan, en la Tabla A, los cálculos necesarios para seleccionar la potencia que transforma a la serie y la hace estable en varianza. Para simplificar, se proponen cinco posibles valores para obtener el valor óptimo de $\lambda$ : $-1,-0.5$, $0,0.5$, y 1 .

Tabla A. Obtención de la potencia óptima $\lambda$ para estabilizar la varianza de $b_{t}$ Potencia Lamda

\begin{tabular}{ccccc}
-1 & -0.5 & 0 & 0.5 & 1 \\
\hline 0.373212126 & 0.178974622 & 0.085827639 & 0.041158816 & 0.019737793 \\
0.288850768 & 0.15193599 & 0.079918587 & 0.042037311 & 0.022111696 \\
0.186406251 & 0.104176753 & 0.058221201 & 0.032538049 & 0.01818452 \\
0.092490146 & 0.049244864 & 0.026219622 & 0.013960208 & 0.007432884 \\
0.149165001 & 0.077454504 & 0.040218551 & 0.020883638 & 0.01084391 \\
0.057044775 & 0.028826722 & 0.014567151 & 0.007361291 & 0.003719918 \\
0.134453746 & 0.069296478 & 0.035714898 & 0.018407198 & 0.009486935 \\
0.583991235 & 0.259200384 & 0.115044259 & 0.051061582 & 0.022663322 \\
0.883485289 & 0.377182152 & 0.161028573 & 0.068747159 & 0.029349896 \\
0.642819783 & 0.298904031 & 0.138987041 & 0.064627424 & 0.030051031 \\
0.142121295 & 0.069894251 & 0.0343735 & 0.016904645 & 0.008313585 \\
0.257637491 & 0.129929783 & 0.065525202 & 0.033045172 & 0.01666509 \\
0.176387587 & 0.093810944 & 0.049892928 & 0.026535329 & 0.014112695 \\
0.074717972 & 0.039175939 & 0.02054063 & 0.010769812 & 0.005646801 \\
0.146326355 & 0.07978964 & 0.043508134 & 0.023724355 & 0.012936547 \\
0.521042665 & 0.272738931 & 0.142764747 & 0.074729974 & 0.039117282 \\
0.84438629 & 0.33898751 & 0.136090002 & 0.054634723 & 0.021933668 \\
0.162870825 & 0.077524027 & 0.036900254 & 0.017563958 & 0.008360176 \\
0.165103142 & 0.079888553 & 0.03865572 & 0.018704366 & 0.009050492 \\
0.299179748 & 0.134550696 & 0.060511749 & 0.027214068 & 0.012239036 \\
0.154576985 & 0.062756043 & 0.025478055 & 0.010343725 & 0.004199404 \\
0.233650663 & 0.094310202 & 0.038067148 & 0.015365334 & 0.006202027 \\
0.322661946 & 0.139595445 & 0.060394132 & 0.026128726 & 0.01130425 \\
\hline 0.299677482 & 0.139484716 & 0.065584771 & 0.031149864 & $0.01494186^{1}$ \\
\hline 0.236826689 & 0.100434729 & 0.043657794 & 0.019646712 & $0.00922197^{2}$ \\
\hline 0.79027189 & 0.720041108 & 0.665669699 & 0.630715822 & $0.61718992^{3}$ \\
\hline & & & &
\end{tabular}

$1=$ Media $; 2=\mathrm{DS}$ (Desviación estándar) y $3=\mathrm{CV}$ (Coeficiente de variación). Donde el criterio es escoger el valor de la potencia de Lamda que arroje el menor CV.

La estimación del modelo AR(1) y la construcción del intervalo de confianza para el parámetro fueron obtenidos con el paquete estadístico $\mathrm{E}-\mathrm{Views}{ }^{\complement}$. . Los resultados son: 
Tabla B. Estimación del modelo AR(1)

Dependent Variable: B

Method: Least Squares

Date: 05/19/04 Time: 22:18

Sample(adjusted): 1980:2 2003:1

Included observations: 92 after adjusting endpoints

Convergence achieved after 3 iterations

\begin{tabular}{ccccc}
\hline Variable & Coefficient & Std. Error & t-Statistic & Prob. \\
\hline C & 0.233430 & 0.022380 & 10.43046 & 0.0000 \\
AR(1) & 0.902085 & 0.046355 & 19.46040 & 0.0000 \\
\hline R-squared & 0.807982 & Mean dependent var & 0.2367252 \\
Adjusted R-squared & 0.805849 & S.D. dependent var & 0.047558 \\
S.E. of regression & 0.020955 & Akaike info criterion & -4.871336 \\
Sum squared resid & 0.039522 & Schwarz criterion & -4.816515 \\
Log likelihood & 226.0815 & F-statistic & 378.7072 \\
Durbin-Watson stat & 1.973065 & Prob(F-statistic) & 0.000000 \\
\hline Inverted AR Roots & .90 & & \\
\hline
\end{tabular}

\section{Bibliografía}

Bencivenga, V. and B. Smith (1991). Financial Intermediation and Endogenous Growth. The Review of Economic Studies, 58, pp.195-209.

Benhabib, J. and M. Spiegel (2001). The Role of Financial Development in Growth and Investment. Journal of Economic Growth, 5, pp. 341-360.

Guerrero, V. (1993). Análisis Estadístico de Series de Tiempo Económicas. 1era ed., UAM, México.

Hamilton, J. D. (1994). Time Series Análisis. 1st ed., Princeton University Press, New Jersey. 